\title{
Les termes, les objets, les notions et les points de vue de la linguistique : problèmes de corrélation
}

\author{
Denis Zolotukhin ${ }^{1}$ \\ ${ }^{1}$ Université Pédagogique d’État de Moscou, Russie.
}

Résumé. La présente contribution vise à analyser quelques problèmes structurels et épistémologiques de la terminologie linguistique dans le français. Par le biais d'une approche sémantique, nous mettons en évidence les particularités du sens terminologique, sa corrélation avec la notion scientifique et sa dépendance de l'objet de recherche linguistique, c'est-àdire du fait langagier de départ. L'analyse des cas précis des termes phrase, sémantème, langue parmi d'autres met en relief l'importance du « jeu» des points de vue dans le domaine linguistique contribuant à la modification de l'extension et de l'intension des sens. En guise de la résolution des problèmes, nous recourrons aux approches de la terminologie générale ainsi qu'à l'application des théories épistémologiques permettant d'introduire une distinction entre des concepts de terminologie et de système terminologique, de termes et de prétermes, de termes aspectuels et de termes synthétisants, de relations intersystémiques et de relations intrasystémiques.

\begin{abstract}
Linguistic terms, objects, notions and points of view: correlation issues. The present work aims to analyze some structural and epistemological issues of linguistic terminology in French. Through a semantic approach, we highlight the peculiarities of the terminological meaning, its correlation with the scientific notion and its dependence on the object of linguistic research - on the linguistic fact itself. The case study of the terms phrase, sémantème, langue among others shows the importance of the "play" of points of view in the linguistic domain contributing to the modification of the meaning extension and intension. In order to solve revealed problems, we use the approaches of general terminology as well as the application of epistemological theories to distinguish between concepts of terminology and terminological system, terms and preterms, aspectual terms and synthesizing terms, intersystemic and intrasystemic relations.
\end{abstract}

\section{Introduction}

L'étude de la terminologie linguistique peut s'effectuer sur différents niveaux d'abstraction. Il est possible d'appliquer une approche soit sémasiologique soit onomasiologique - commencer par des unités lexicales, démontrer des particularités de leurs relations ou bien recourir à l'analyse des notions décrites. Mais les deux approches nous ramènent inévitablement au-delà des unités en tant que telles, au-delà du métalangage et même au-delà de la langue française car nous serons toujours confrontés au faisceau de problèmes terminologiques - à l'objet de recherche et au point de vue sur cet objet. Ainsi, le schéma complexe de la production métalinguistique est formé par plusieurs éléments mêlant 
des unités linguistiques à des unités non-linguistiques et, comme le déclare F. Neveu :

cela n'est pas sans conséquences sur l'activité terminologique, notamment $<\ldots>$ pour ce qui est du développement du nombre des métatermes et de leur variabilité définitionnelle et formelle. Les " objets " de la science linguistique sont des objets de pensée (Neveu 2008).

Dans la présente contribution, nous tenons à illustrer ce « cercle vicieux » qui mène tout linguiste de la réalité linguistique (langue-objet) au domaine de la pensée (objets abstraits, notions scientifiques) pour qu'il revienne au bout du compte à la réalité linguistique (langueinstrument) dans laquelle et par le biais de laquelle il utilise et produit des termes.

\section{De l'objet linguistique au terme}

Le terme linguistique est une unité lexicale du métalangage désignant une notion scientifique d'une ou de plusieurs théories linguistiques. On est loin ici de la considération des termes comme des « étiquettes » servant à dénommer des notions, comme le suggère, par exemple, G. Lazard (1999). Tout terme est une entité, un signe linguistique se composant d'un signifié et d'un signifiant (Depecker 2002) d'où viennent de nombreux problèmes sémantiques. Ainsi, une notion scientifique est exprimée par un sens terminologique se composant de son extension (des limites d'un objet abstrait résultant de la combinaison et de la généralisation de nombreux objets spécifiques) et de son intension (des caractéristiques et des aspects d'un objet décrit résultant de l'interprétation scientifique de cet objet). La construction de l'intension d'un terme implique la mise en valeur des caractéristiques notionnelles correspondant à un point de vue à partir duquel l'objet (une extension sélectionnée) est considéré par un chercheur. Par conséquent, c'est au niveau de l'intension que l'on trouve la différence entre le terme et l'unité lexicale de la langue ordinaire. Ainsi, un objet spécifique (un fragment de la réalité linguistique, un fait linguistique) peut être considéré à partir de différents points de vue. S'il existe une compréhension ordinaire de l'objet, nous parlons du sens couramment utilisé d'une unité lexicale de la langue ordinaire. C'est ce dont rend compte le schéma suivant :

$$
\text { objet spécifique } \rightarrow \text { notion ordinaire } \rightarrow \text { sens commun }
$$

Par exemple, en français, le mot phrase possède le sens commun de «tour, expression » (Le Nouveau Petit Robert 2009). Lorsqu'une réflexion scientifique sur le même fait linguistique a lieu, c'est l'intension de la notion qui change. De nouveaux aspects et de nouvelles caractéristiques de l'objet sont révélés sans que, en règle générale, ses limites ne soient modifiées car il s'agit toujours de la même extension. Ainsi, la notion scientifique est créée, le sens terminologique est formé et l'unité lexicale devient terme :

\section{objet spécifique $\rightarrow$ notion scientifique $\rightarrow$ sens terminologique}

Un tel schéma se justifie dans la théorie du linguiste A. Potebnia qui a distingué le sens le plus « lointain » du sens le plus « proche » des unités lexicales. Le sens le plus proche correspond au contenu central d'un mot particulier. C'est le sens faisant partie du système de la langue ordinaire, il est alors accessible à tout sujet parlant cette langue. Le sens le plus lointain porte une information scientifique contenant des caractéristiques extralinguistiques. C'est pour cette raison que le sens le plus lointain est accessible plutôt aux communautés scientifiques, il représente le sens terminologique (Kobozeva 2009, p. 63). Suite à ce schéma, la phrase reçoit une définition scientifique construisant un sens « lointain » (sens terminologique) de la même unité : 
grammaticaux) et que l'émetteur et le récepteur considèrent comme un énoncé complet; unité minimale de communication (Le Nouveau Petit Robert 2009).

Il est indispensable de noter que dans ce cas, l'unité lexicale devient un terme linguistique parce que l'objet est décrit notamment du point de vue de la science du langage. La même unité lexicale acquiert le statut de terme musical si la phrase est définie comme :

partie d'une ligne mélodique ou d'une idée musicale naturellement délimitée, significative du point de vue de la déclamation, de l'articulation et de la respiration $<\ldots>$ » (TLFi).

En même temps, le changement de point de vue linguistique en point de vue musical contribue à un changement non seulement de l'intension, mais aussi de l'extension du sens puisque la musique et la linguistique peuvent définir les limites de ce que le terme phrase signifie.

L'un des problèmes de l'étude de la sémantique des termes linguistiques est qu'au sein de la linguistique française, il est impossible de parler d'un seul point de vue. La présence d'un grand nombre d'écoles et de disciplines linguistiques mène au développement d'une forte variabilité ou du polymorphisme des termes (Shelov 2003, pp. 67-87). F. de Saussure a attiré l'attention sur ce phénomène en déclarant qu'en linguistique, un objet de départ n'existe pas du tout, l'objet est toujours créé par un point de vue choisi : "ily a d'abord les points de vue à l'aide desquels on CRÉE secondairement les choses » (Saussure 2002, p. 200). Dans l'étude des faits linguistiques, des chercheurs modélisent leur propre objet abstrait qui peut s'avérer différent du fait linguistique de départ. Un changement de point de vue modifie également l'objet lui-même. Par conséquent, dans la réflexion scientifique sur les faits linguistiques, on peut observer un schéma suivant :

$$
\text { fait linguistique spécifique } \rightarrow \underset{\text { terme }}{\text { objet abstrait }} \rightarrow \text { notion scientifique } \rightarrow \text { sens du }
$$

\section{Les faits linguistiques - ces objets continus et polyaspectuels}

La difficulté de la création et de la description des termes linguistiques est également causée par le tout premier composant de la chaîne présentée ci-dessus, c'est-à- dire par le fait linguistique défini par V. Gak (1998) comme continu et polyaspectuel. Le caractère continu des faits linguistiques se manifeste dans le manque des « lignes de séparation », des frontières nettes entre les objets (Gak 1998, p. 22). Ce caractère permet d'établir arbitrairement des limites de l'objet et de former l'extension d'une notion et d'un sens terminologique de différentes manières.

Nous pouvons reprendre ici l'exemple du terme phrase. Selon A. Brachet, une phrase s'étend d'un point à un autre : «Elle [phrase] est ordinairement comprise entre deux points » (Brachet 1895, cité dans TLFi). L'idée du «flou » des limites de l'objet se retrouve dans une déclaration qui jette un doute sur la nécessité de prendre en compte la ponctuation lors de la définition des limites d'une phrase :

La ponctuation n'a rien de rigoureux. Combien faut-il voir de phrases dans un énoncé comme celui-ci : Des Chinois arrivèrent aussi. Si bien qu'au bout de quelques mois Cox-City comptait près de cinq mille habitants " (Chevalier 1964, cité dans TLFi).

Les limites de l'objet dépendent toujours des objets adjacents ou similaires. Ainsi, l'extension du sens de phrase change avec l'introduction du terme énoncé : 
la phrase relève de la compétence, et l'énoncé de la performance $<$... $>$ deux énoncés sont des occurrences distinctes d'une même phrase (Ruwet 1967, cité dans TLFi).

En raison de l'apparition de différentes extensions, un terme peut commencer à désigner progressivement deux ou plusieurs objets aux limites différentes, ce qui contribue à la formation d'une ambiguïté terminologique et à la désintégration de la sémantique du terme soit en plusieurs sens séparés (le terme polysémique), soit en homonymes. Un tel mécanisme permettant de créer un objet et un terme correspondant est bien démontré par les unités utilisées pour désigner les domaines scientifiques et les objets de recherche. Par exemple, deux extensions apparaissent dans la sémantique du terme syntaxe:

1) Étude des relations entre les unités du syntagme;

2) Relations entre les unités du syntagme (Le Nouveau Petit Robert 2009).

Dans ce cas, le point de vue syntaxique crée un objet syntaxique, alors que l'existence de l'un sans l'autre est impossible car lorsque ce point de vue est supprimé, un objet aussi abstrait que des relations syntagmatiques disparaîtra et on n'aura qu'un nombre infini de faits linguistiques sous la forme de combinaisons illimitées.

Les points de vue morphologiques, sémantiques, étymologiques et d'autres peuvent être orientés vers des objets spécifiques à la base desquels de nouveaux objets abstraits sont formés et les termes correspondants (morphologie, sémantique, étymologie, etc.) comporteront deux sens : (1) point de vue/ étude/ discipline et (2) objet.

Notons que F. de Saussure, conscient de la difficulté de séparer un objet d'un point de vue, a toujours essayé de distinguer ces termes. Ainsi, les termes synchronie et diachronie ne sont jamais utilisés dans ses manuscrits, le linguiste ne les repend qu'à l'oral lors des cours devant ses étudiants. Pour désigner la synchronie et la diachronie en tant que points de vue et en tant qu'objets, F. de Saussure utilise des combinaisons tout à fait différentes :

1) point de vue, étude, aspect $<$... synchronique / diachronique (la synchronie et la diachronie comme approches) ;

2) objet, axe $\langle\ldots>$ synchronique / diachronique (la synchronie et la diachronie comme états statique ou dynamique de la langue) (Saussure 2002).

Une telle interaction entre une discipline ou une méthode et son objet semble être évidente et même logique parce que dans ce cas, un sens terminologique reste indissolublement lié à l'autre, les deux continuent à fonctionner et peuvent être repris dans le même texte.

Ce n'est pas le cas pour les termes qui nous mènent à un grand nombre de difficultés en raison du «conflit » entre deux ou plusieurs points de vue. Ainsi, on observe la création des objets et des notions dont les limites s'entrecroisent et se superposent. Par exemple, A.-J. Greimas définit le terme sémantème comme «investissement sémantique d'un morphème ou d'un énoncé » (Greimas 1980, p. 325). Le linguiste utilise ce terme pour faire référence au noyau sémantique combiné aux sèmes contextuels (variations de sens). Pour B. Poitiers, le terme sémantème désigne un groupe de sèmes distinct : "ensemble de sèmes spécifiques de l'unité considérée » (cité dans Tsybova 2002, p. 27). Dans la théorie de Ch. Bally le terme sémantème ne désigne pas le contenu ou une partie du contenu de l'unité, mais un signe entier exprimant une signification purement lexicale (Bally 1932, p. 64). Par conséquent, dans ces théories linguistiques, le terme sémantème est corrélé avec au moins 3 notions :

1) Ensemble de sèmes ;

2) Ensemble de sèmes spécifiques ;

3) Signe à une signification lexicale.

D'une part, il y a trois sens terminologiques entre lesquels s'établissent des relations métonymiques assez stables. Ainsi, dans le cas des sens (1) et (2), il s'agit de la synecdoque basée sur le lien entre une entité (1-ensemble de sèmes) et une de ses parties (2-ensemble de sèmes spécifiques). Le sens (3) est également associé aux sens (1) et (2) car il s'agit d'un tout (d'un signe entier) par rapport à une partie (le contenu d'un signe).

Ces sens étroitement liés pourraient être inclus dans le même système sémantique d'un seul terme sémantème qui serait alors polysémique. Mais pour des raisons pratiques, il est 
indispensable de considérer ces trois sémantèmes comme trois termes différents : sémantème comme ensemble de sèmes, sémantème comme ensemble de sèmes spécifiques, sémantème comme signe à une signification lexicale. S'agit-il alors de l'homonymie terminologique ? Il s'avère évident que ce n'est pas la même homonymie que dans le cas, par exemple, des termes langue en linguistique et langue en physiologie car le lien entre les deux objets et entre les deux sens est aujourd'hui beaucoup plus éloigné qu'entre ces trois sens de sémantème. De plus, il s'agit des deux sphères scientifiques différentes, ce qui nous permet de parler des deux terminologies - celle de la linguistique et celle de la physiologie, ou de deux " souslangages » différents si nous reprenons l'approche de P. Swiggers (1999). Mais même quand nous nous adressons au système terminologique en linguistique à part, nous y découvrons toujours deux homonymes : langue en sémantique (langue comme système de signes) et en phonétique (langue comme organe situé dans la cavité buccale).

La terminologie en linguistique devrait être alors divisée en sous-terminologies selon le nombre des domaines linguistiques. Le problème est qu'au sein de chaque sousterminologie, il existe, comme nous l'avons déjà mentionné ci-dessus, de nombreuses branches et écoles ayant chacune sa propre terminologie. C'est le cas de notre exemple de sémantème. Une telle terminologie peut même être créée et utilisée par un seul linguiste et ne jamais sortir de ses textes manuscrits ou publiés.

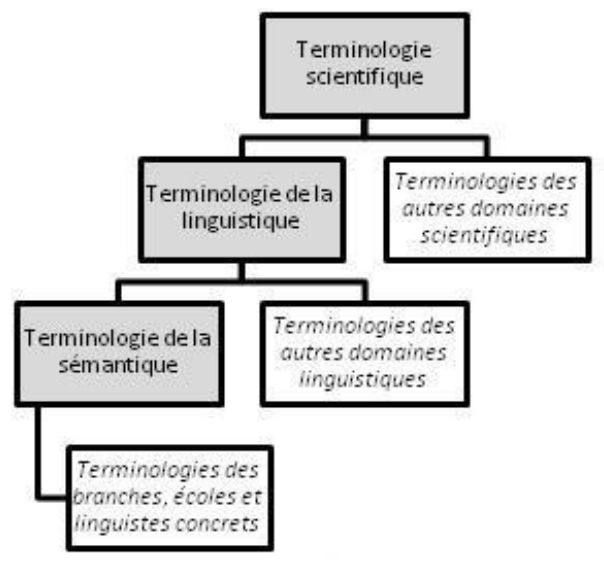

Fig. 1. Hiérarchie des terminologies.

De ce point de vue, il est essentiel que non seulement les lexicographes mais aussi tout chercheur se servant des éléments de la terminologie linguistique établisse deux formes de relations entre les termes :

1) des relations intersystémiques

2) des relations intrasystémiques.

Ainsi, le terme de sémantème possède des homonymes intersystémiques, mais n'a pas d'homonymes intrasystémiques si nous l'analysons au sein des terminologies particulières de chaque auteur. Par contre, il obtient des homonymes intrasystémiques dans la terminologie de la sémantique. La considération des relations des termes est aussi importante car elles s'établissent entre des éléments de tous les niveaux, même avec des unités lexicales de la langue ordinaire car il s'agit toujours d'un sous-langage qui, selon

P. Swiggers, représente « un binôme comprenant un lexique $<_{\ldots} . .>$ et un ensemble de règles de formation lexicale et de construction syntaxique " du langage super-ordonné (Swiggers 1999, p. 31).

Le caractère continu de l'objet linguistique cause ainsi des problèmes de la délimitation des termes et, par conséquent, la classification sémantique des éléments dans la terminologie. Néanmoins, ces problèmes peuvent se mettre en évidence étant donné que les limites des objets, bien que floues, sont relativement saisissables. Il n'en va pas de même pour les cas où les chercheurs, de façon implicite ou explicite, touchent à l'intension des notions décrites. 
Nous y faisons face au caractère polyaspectuel de l'objet linguistique ayant de nombreux aspects, manifestations et liens intersystémiques et intrasystémiques (Gak 1998, p. 24).

Mais tout d'abord, il serait juste de mentionner que ce caractère polyaspectuel nous permet de nous focaliser sur un certain détail, d'approfondir au mieux la connaissance déjà existante lors de la réalisation d'une activité scientifique, c'est-à-dire de mettre en évidence de nouveaux aspects et caractéristiques de l'objet, de modifier le contenu de la notion scientifique et de former une nouvelle intension du sens terminologique avec une extension pertinente - sans changer les limites de l'objet. Ce caractère a été bien démontré par le linguiste $\mathrm{M}$. Blokh qui prend le terme russe slovo (mot, lexème) à titre d'exemple : le mot en tant que fait linguistique peut avoir au moins sept aspects, ou « incarnations » (phonémique, catégorique, etc.) (Blokh 2012, p. 6).

Dans la terminologie française, le caractère polyaspectuel de l'objet est également démontré par la sémantique du terme phrase. Ainsi, dans la grammaire classique, la phrase est définie plutôt superficiellement et a le sens d'un «ensemble de mots qui a un sens complet et qui va d'un point à un autre » (Hamon 1964, cité dans TLFi). Le structuralisme prend en compte le lien entre la phrase et la parole, ainsi que sa composition structurelle et ses relations internes : "le type par excellence du syntagme <...> appartient à la parole, non à la langue »(Saussure 1997, cité dans TLFi), "l'énoncé dont tous les éléments se rattachent à un prédicat unique ou à plusieurs prédicats coordonnés » (Martinet 1969, cité dans TLFi). La grammaire générative se concentre sur une relation entre les phrases et les situations de communication verbale :

une entité linguistique abstraite, purement théorique, en l'occurrence un ensemble de mots combinés selon les règles de la syntaxe, ensemble pris hors de toute situation de discours, ce que produit un locuteur, ce qu'entend un auditeur (Ducrot 1980, cité dans TLFi).

Les représentants des écoles citées semblent être d'accord sur l'extension de la notion de phrase - ils établissent les mêmes limites de l'objet dont ils s'approchent (ensemble de mots ... d'un point à un autre; syntagme ; un prédicat unique ou à plusieurs prédicats), mais chacun interprète cet objet de son propre point de vue (des points de vue de la grammaire classique, du structuralisme ou de la grammaire générative) se concentrant sur un des aspects de la phrase.

Il existe d'autres termes français incluant une intension variable en fonction d'un aspect choisi de l'objet de départ. Les termes de la linguistique générale sont sujets à la plus grande variabilité puisque c'est dans ce domaine linguistique que nous retrouvons des notions très complexes : termes langage, langue, parole, signe linguistique, etc. Par exemple, dans la structure sémantique du terme langue utilisée par différents auteurs pour indiquer l'objet central de la recherche linguistique, on peut relever des archisèmes /système/, /instrument/, /institution/, /ensemble de moyens/, /manifestation/, etc. qui sont tous une sorte d'expression d'une approche choisie et peuvent former un sens à part dans la perspective de se transformer en un terme isolé.

\section{En guise de la synthèse}

Le nombre de particularités des termes de la linguistique française mises en relief dans la présente recherche nous fait poser la question suivante : les unités lexicales considérées comme termes linguistiques et utilisées dans les textes scientifiques sont-elles toutes de vrais termes, au sens strict ? La réponse est négative si des critères assez concrets sont pris en compte car le terme doit refléter le résultat final de la spécialisation d'une unité lexicale - il $\mathrm{y}$ a une transition progressive d'un terme "prématuré » à un vrai terme scientifique. L'étymologie même du mot terme formé à partir du terminus latin (fin, but final ou frontière, limite) et utilisé au XIe siècle pour indiquer l'heure à laquelle tout événement attendu se produit (Le Nouveau Petit Robert 2009) démontre bien ce critère de spécialisation progressive. Depuis le XIVe siècle le terme est interprété en français comme une unité définissant les limites du sens (Rey 1979, 15-20 p.), c'est-à-dire des limites rigoureuses et 
non floues. C'est pour cette même raison que $\mathrm{M}$. Blokh recourt à la notion de "rigorème » (rus. rigorema) pour désigner la sémantique d'un vrai terme (Blokh 2012, p. 10). Il est aussi à noter que selon F. de Saussure, tout mot a un certain rapport avec d'autres mots et il n'existe que par rapport à ces autres mots, c'est-à-dire il est un élément du système de la langue, ou un terme (Saussure 2002, p. 351). Il en résulte que le terme en synchronie se caractérise par une structure sémantique stable développée et par la présence des rapports établis avec d'autres termes du système qui en définissent clairement les limites. Du point de vue diachronique, le terme est lui-même une limite de la spécialisation d'une unité lexicale.

Ainsi, la plupart des termes linguistiques d'aujourd'hui se trouvent en voie de spécialisation et ne peuvent pas être considérés comme termes au sens strict - ils sont plutôt des éléments du continuum entre un terme et une unité lexicale du langage commun. Ces éléments de la terminologie de la linguistique française doivent être dénommés et nous pouvons nous servir ici de la désignation préterme (rus. predtermin) proposée par le terminologue russe V. Leytchik (2012).

À cet égard, l'ensemble des unités terminologiques de la linguistique française se compose aujourd'hui des termes et des prétermes. Si on reprend la théorie de V. Leytchik, il s'agit de la terminologie de la linguistique française - un ensemble partiellement ordonné de termes et de prétermes qui n'est pas caractérisé par une aussi stricte cohérence vu le nombre de points de vue y coexistant. L'évolution de cette terminologie doit mener à la formation d'un ensemble intégral et ordonné de termes, c'est-à-dire à la construction du système terminologique (rus. terminosistema, Leytchik 2012) de la linguistique française.

Ce processus de systématisation demande un travail scrupuleux sur chaque unité terminologique, d'où vient la nécessité de la réinterprétation des termes et des notions existants par le biais de l'analyse épistémologique. Ainsi, le pluralisme des points de vue affectant l'objet en cours de construction attache une importance particulière à la mise en évidence de deux types de conceptions linguistiques dans l'histoire de la science du langage (Zubkova 2002, p. 20-22).

Premièrement, ce sont des conceptions aspectuelles décrivant un aspect particulier de l'objet, ce qui permet d'analyser cet aspect en détail, de développer des méthodes spéciales appropriées. Cela contribue en même temps à un grand nombre de contradictions entre les conceptions aspectuelles. Comparez, par exemple, la théorie des néogrammairiens à la théorie néolinguistique. La théorie de F. de Saussure peut alors être considérée comme aspectuelle car elle exclut le «jeu» des points de vue sur la langue en l'examinant « envisagée en elle-même et pour elle-même » (Saussure 1995, p. 305).

Deuxièmement, il existe des conceptions synthétisantes tenant compte de tous les aspects du phénomène décrit. Les conceptions synthétisantes tiennent à présenter toute cette ampleur des caractéristiques, des traits, des « côtés » de l'objet continu et polyaspectuel. C'est le cas, par exemple, de la théorie de G. Guillaume dont les recherches ont mis en valeur les liens entre la langue, la parole, les phénomènes extérieurs et psychiques, et de E. Benveniste démontrant l'unité de la pensée et du langage.

Cette classification des théories peut être transférée au domaine de la terminologie linguistique dans le cadre de laquelle se distinguent les termes aspectuels dont le sens n'exprime que de certaines caractéristiques notionnelles de l'objet, et les termes synthétisants unissant dans leur sens tous les aspects et limites de l'objet établis au moment précis de l'histoire de la science du langage. Les exemples énumérés ci-dessus permettent de constater que dans les textes des linguistes francophones, les termes phrase et sémantème font partie des termes aspectuels, pendant que le terme langue est plutôt synthétisant.

À première vue, les termes synthétisants représentent un avantage car ce sont effectivement ces unités qui fournissent des informations complètes sur un fait linguistique. La perspective de toute recherche linguistique, suivant la logique de la pensée scientifique, devrait consister tout d'abord à créer un ensemble de termes aspectuels dont le nombre devrait être déterminé par le nombre de variantes des limites de l'objet, ainsi que par le nombre d'aspects éclairés dans celui-ci. Il faudrait ensuite procéder à la création des termes synthétisants dont la sémantique n'exprimerait que des variantes approuvées par la communauté scientifique. Cependant, une telle évolution de la terminologie linguistique 
supposant la création progressive des termes synthétisants reste controversée et contribue à une discussion supplémentaire si nous tenons compte de la déclaration faite par F. de Saussure à propos du polymorphisme en linguistique :

Il n'y a aucun terme définissable et valable hors d'un point de vue précis, par suite de l'absence totale d'êtres linguistiques donnés en soi $<\ldots>$ Il n'est plus permis de faire usage d'un terme emprunté au point de vue A si le on passe au point de vue $\mathrm{B}<\ldots>$ En linguistique on peut se demander si le point de vue où on envisage la chose n'est pas toute la chose ou s'il n'y a jamais eu autre chose que nos points de vue indéfiniment multipliables (Saussure 2002, p. 67, p. 81).

Cette thèse correspond absolument au principe de parcimonie selon lequel il faut «utiliser le nombre minimal de termes requis par la description du domaine » (Swiggers 1999, p. 31, Neveu 2008, p. 89).

\section{Conclusion}

Les difficultés auxquelles nous nous trouvons confrontés en tant que linguistes au sens large, c'est-à-dire des chercheurs se servant et créant des termes français, et en tant que terminologues tâchant de décrire et classifier les unités terminologiques, sont ainsi étroitement liées aux trois sphères de différents niveaux formant un cercle :

sphère linguistique 1 où se trouvent des objets de nos recherches - des faits linguistiques polyaspectuels et continus ;

sphère mentale où nous construisons des objets abstraits en généralisant tout ce que nous relevons dans la sphère linguistique et en établissant arbitrairement des limites et des aspects de ces objets à la base d'un point de vue choisi - c'est ainsi que nous créons des notions scientifiques ;

sphère linguistique 2 où se trouvent des termes dont le signifié inclut l'extension corrélée avec des limites établies et l'intension corrélée avec un aspect (termes aspectuels) ou plusieurs aspects (termes synthétisants) étudiés. C'est dans cette sphère que nous procédons à un vrai travail lexicologique et même lexicographique. Nous espérons que les notions présentées et décrites ci-dessus pourront servir d'outils nécessaires à l'analyse sémantique des éléments dans la terminologie linguistique française.

Le parcours circulaire des linguistes suppose ainsi le travail avec des termes et des prétermes, ce qui contribue progressivement à l'évolution de la terminologie linguistique et à sa transformation en système terminologique plus ou moins stable et composé des termes aspectuels et synthétisants entre lesquels s'établissent des relations intersystémiques et intrasystémiques.

Le problème à résoudre consiste à décider jusqu'à quel point nous devons mener l'évolution des termes. Un vrai système terminologique en linguistique doit-il absolument correspondre à ce que nous observons dans le domaine des sciences exactes et techniques ? Les quantités du langage et leurs rapports sont-ils " régulièrement exprimables de leur nature fondamentale, par des formules mathématiques » (Saussure 2002, p. 206) ? La présente étude des exemples démontre que ni du point de vue sémantique, ni du point de vue épistémologique, nous ne pouvons parler de la cohérence ou de l'exactitude mathématique dans la linguistique. Il s'agit d'un système spécifique, qui se développe selon ses propres lois et mécanismes, pour servir d'outil à cette science si particulière qui traite du langage.

\section{REFERENCES BIBLIOGRAPHIQUES}

Bally C. (1932). Linguistique générale et linguistique française. Paris : Leroux.

Blokh M. (2012). « Slovo i ego voploshheniya », Vestnik Irkutskogo gosudarstvennogo lingvisticheskogo universiteta, Irkutsk, T.1, 17, pp. 6-15.

Depecker L. (2002). Entre signe et concept. Éléments de terminologie générale. Paris : Presses Sorbonne 
Nouvelle.

Gak V. (1998). Yazykovye preobrazovaniya. Moscow : Shkola Yazyki russkoj kultury.

Geeraerts D. (2010). Theories of lexical semantics. Oxford : Oxford University Press.

Greimas A. J.(1980). Sémiotique : Dictionnaire raisonné de la théorie du langage. Paris : Classiques Hachette, Hachette Université.

Kobozeva I. (2009). Lingvisticheskaja semantika. Moscow : Knizhnyj dom Librokom.

Lazard G. (1999). « Pour une terminologie rigoureuse », in A. Lemaréchal (dir.), La Terminologie linguistique, Mémoires de la Société de Linguistique de Paris, nouvelle série, tome VI, Paris, Peeters, pp. 111-133.

Le Nouveau Petit Robert de langue française (2009, version 3.2). Paris : Dictionnaires le Robert (CDROM).

Leychik V. (2012). Terminovedenie : predmet, metody, struktura. Moscow : Librokom.

Neveu F. (2008). « Pour une description terminographique des sciences du langage », in John

Humbley (ed.) Cahier du CIEL 2007-2008, «Aspects de la recherche en langues de spécialité », Université Paris VII, UFR EILA, pp. 87-104.

Rey A. (1979). La terminologie, noms et notions. Paris : Presse Universitaire de France. Saussure, Ferdinand de (1995). Cours de linguistique générale, Publ. par Ch. Bally et A. Sechehaye avec la collaboration de A. Riedlinger, Ed. critique prép. par Tullio de Mauro. Paris : Gallimard. 
Saussure F.de (2002). Écrits de linguistique générale. Établis et édités par Simon Bouquet et Rudolf Engler avec la collaboration d'Antoinette Weil. Paris : Gallimard.

Shelov S. (2008). "Terminologicheskie polya i terminologicheskaya organizatsiya terminologii ", Strukturnaya i prikladnaya lingvistika, Vypusk 7, Mezhvuzovskiy sbornik, Saint Petersburg, pp. 211233.

Swiggers P. (1999). "Pour une systématique de la terminologie linguistique : considérations historiographiques, méthodologiques et épistémologiques », in A. Lemaréchal (dir.), La Terminologie linguistique, Mémoires de la Société de Linguistique de Paris, nouvelle série, tome VI, Paris, Peeters, pp. 11-49.

Trésor de la langue Française informatisé. ATILF - CNRS \& Université de Lorraine [en ligne].

Disponible sur : http://www.atilf.fr/tlfi (consulté le 01.09.2019).

Tsybova I. (2002). Essai de lexicologie française. Szczecin : Wydawnictwo naukowe Uniwersytetu Szczecinskiego.

Zubkova L. (2002). Obshaya teoriya yazika v razvitii. Moscow : RUDN. 